JOURNAL OF THE

AMERICAN MATHEMATICAL SOCIETY

Volume 10, Number 2, April 1997, Pages 445-466

S 0894-0347(97)00233-6

\title{
MATRIX $A_{p}$ WEIGHTS VIA $S$-FUNCTIONS
}

\author{
A. VOLBERG
}

\section{INTRODUCTION}

The statement of the problem. In this paper we study weighted norm inequalities with matrix valued weights. Namely, let $W$ be a $d \times d$ matrix weight, i.e. a $L^{1}$-function whose values are selfadjoint nonnegative $d \times d$ matrices. We suppose that the weight $W$ is defined on the unit circle $\mathbb{T}=\{z \in \mathbb{C}:|z|=1\}$ or the real line $\mathbb{R}$.

Let $L^{p}=L^{p}\left(\mathbb{C}^{d}\right)$ be the space of all measurable vector functions on $\mathbb{T}$ whose $\mathbb{C}^{d}$ norm is summable to the power of $p$. Let $H^{p}=H^{p}\left(\mathbb{C}^{d}\right)$ be the corresponding Hardy space of analytic functions, and let $P_{+}$be projection in $L^{p}$ onto $H^{p}$ annihilating antianalytic functions in $L^{p}$ which vanish at the origin. Let $H$ denote the Hilbert transform, $H=-i P_{+}+i\left(I-P_{+}\right)$. Clearly, $P_{+}, P_{-} \stackrel{\text { def }}{=} I-P_{+}$, and $H$ act as usual operators $P_{+}, P_{-}$, and $H$ coordinatewise if we fix for all $t \in \mathbb{T}$ the same coordinate basis in $\mathbb{C}^{d}$.

We are interested in the conditions on $W$ under which the following weighted norm inequality for operator $H$ holds (say for all $f \in L^{p} \cap L^{\infty}$ ):

$$
\int_{\mathbb{T}}\left(W(t)^{2 / p} H f(t), H f(t)\right)^{p / 2} d m(t) \leq C \int_{\mathbb{T}}\left(W(t)^{2 / p} f(t), f(t)\right)^{p / 2} d m(t) .
$$

Clearly this inequality is equivalent to the same inequality for $P_{+}$(with another constant). Here $m$ denotes Lebesgue measure on $\mathbb{T}$, and $W(t)^{2 / p}$ stands for the operator power of nonnegative selfadjoint operator $W(t)$. If we define a weighted space $L^{p}(W)$ as the space of all measurable $\mathbb{C}^{d}$-valued functions on $\mathbb{T}$ satisfying

$$
\|f\|_{L^{p}(W)}^{p} \stackrel{\text { def }}{=} \int_{\mathbb{T}}\left(W(t)^{2 / p} f(t), f(t)\right)^{p / 2} d t<\infty
$$

(of course we should factorize it over the subspace of functions of norm 0 ), then the last inequality means that $H$ (or, equivalently, $P_{+}$) is a bounded operator in $L^{p}(W)$. The notation is chosen in such a way that $L^{p}(W)$ becomes a familiar weighted $L^{p}$ space in the scalar situation $d=1$. We consider the range $(1, \infty)$ of $p$.

In the scalar-valued setting $d=1$ the answer is given by the famous HuntMuckenhoupt-Wheeden theorem which says that the Muckenhoupt condition $\left(A_{p}\right)$

$$
\sup _{I}\left(\frac{1}{|I|} \int_{I} W\right)^{1 / p} \cdot\left(\frac{1}{|I|} \int_{I} W^{-q / p}\right)^{1 / q}<\infty
$$

Received by the editors May 24, 1996 and, in revised form, December 2, 1996.

1991 Mathematics Subject Classification. Primary 42B20, 42A50, 47B35.

Key words and phrases. $A_{p}$ weights, matrix-functions, area integrals, singular integrals, Carleson measures, Triebel-Lizorkin spaces.

This work was partially supported by National Science Foundation Grant DMS9622936. 
$(q=p /(p-1)$; supremum is taken over all intervals $)$ is necessary and sufficient in this case.

The main purpose of this paper is to give a matrix analog of $\left(A_{p}\right)$ and to prove that it is necessary and sufficient for the matrix weighted inequality to hold.

In [TV1] the matrix $\left(A_{2}\right)$ condition appeared:

$$
\sup _{I}\left\|\left(\frac{1}{|I|} \int_{I} W\right)^{1 / 2} \cdot\left(\frac{1}{|I|} \int_{I} W^{-1}\right)^{1 / 2}\right\|<\infty .
$$

Here the power $1 / 2$ stands for the operator power of nonnegative operatorsaverages of $W$ in our case. Later $\mathrm{S}$. Treil came up with the conjecture of what the matrix $\left(A_{p}\right)$ should look like.

The matrix $\left(A_{p}\right)$ condition does not have such a simple form if $p$ is not 2 . It cannot be obtained by the combination of averagings and raising to operator powers. It requires the analysis of the geometric meaning of the Muckenhoupt condition $\left(A_{p}\right)$, even though there is no geometry in $\mathbb{C}^{1}$. We explain the matrix $\left(A_{p}\right)$ in the next section. Now let us remark that very few scalar methods are available in the vector situation.

The difficulties. As an illustration of what kind of difficulties one can encounter with the vector case, let us present two very simple examples. It is trivial in the scalar case that if we have an integral operator in $L^{p}(W)$ with positive (scalar) kernel, and we know that an operator with a bigger kernel is bounded, then the original operator is bounded too. This statement (even for scalar kernels) does not hold for weighted $L^{p}$ spaces with matrix weights. Certainly if $W(t)$ can be diagonalized by the same basis for each $t \in \mathbb{T}$, we do not have any difficulties. But this is not the case we are interested in. Another difficulty comes from the fact that while any nonzero nonnegative operator is invertible in $\mathbb{C}^{1}$, this is quite false in $\mathbb{C}^{d}$. For example, suppose we meet the expression (we do meet such expressions while working with matrix weights) $\left(A(I+B)^{-1} x,(I+B)^{-1} x\right)$, where $A$ and $B$ are nonnegative operators, $B \leq I$, and $x$ is a vector in $\mathbb{C}^{d}$. If $A, B, x$ are numbers, the estimate from below $\frac{1}{4} A|x|^{2}$ would follow. But no estimate $\delta\|x\|^{2}, \delta>0$, exists for operators.

The main idea. Nazarov and Treil in $[\mathrm{NT}]$ overcome all the difficulties by using the Bellman function approach (see below). But first a simple idea which was successfully used in [TV1] comes into play. Roughly speaking the idea is as follows. The first step is to choose a good basis in $L^{p}(W)$. It should be good in the sense that:

1) the coefficient space with respect to this basis should be relatively simple;

2) the matrix of the operator $H$ (or $P_{+}$) in this coefficient space should also be relatively simple (desirably as close to a diagonal matrix as possible).

Let $h_{I}$ stand for a Haar function. It turns out that matrix $\left(A_{p}\right)$ is equivalent to the fact that $\left\{h_{I} \mathbb{C}^{d}\right\}_{I \in \mathcal{D}}$ forms an unconditional basis in $L^{p}(W)$. In the scalar situation this is well known (see [FJW]), and the coefficient space is the weighted Tribel-Lizorkin space $f_{p}^{02}$ (see [FJW] and below for the notation).

The Bellman function. The main tool in $[\mathrm{NT}]$ is the use of the Bellman function. Here is what it is. The desired estimates involve the averages of functions and 
weights over dyadic intervals. Consider these averages as "variables". Generally, the estimates one encounters will have the form

$$
\sum_{I \subset J} a_{I} \leq C F_{J}
$$

where $a_{I}$ is a certain (depending on the problem) function of our "variables" (read "averages") corresponding to $I$. Now try to build a pretty concave function $B$ (let us call it the Bellman function of the problem) of our "variables" in such away that:

1) for all $I \in \mathcal{D}, B(I) \leq C F_{I}$,

2) $B(I)-1 / 2\left(B\left(I_{-}\right)+B\left(I_{+}\right)\right) \geq\left|a_{I}\right|$.

Here $B(I)$ stands for a value of function $B$ on "variables" (i.e. averages) corresponding to $I$. The second requirement above is the strong concavity condition. After function $B$ is built, the desired estimate is obtained just by repeatedly applying the concavity. Actually it happens very often that the existense of $B$ is equivalent to the singular integral estimate under consideration.

The function $B$ is closely related to the Burkholder function from [B1], [B2], [B3], [Str], Section 6.3.15, constructed for obtaining the best constants in scalar martingale and singular integral estimates.

The Bellman functions have been used for decades in Control Theory. They can be interpreted as the supremum of price over different plans of distributing the resources. By nature such functions are convex. In our dyadic estimates we have averages (or integrals) over $J$ and we wish to show that whatever the distribution of them over the dyadic sons, grandsons, et cetera is, the price will not exceed $C F_{I}$ provided that the price for distributing from any dyadic $I$ to its dyadic "sons" $I_{-}$ and $I_{+}$is $a_{I}$.

Carleson measures and $A_{\infty}$. In the present paper another approach to proving matrix weighted inequalities is suggested. The observation is that even though many scalar methods are not available now, there is at least one which still manages to survive. We mean the good $\lambda$-inequality, which should be more rigorously called the relative distributional inequality (see [St], pp. 206-209). Let us also remind the reader about the role of $A_{\infty}$ weights. A positive function cannot be much bigger than its average over $I$ on a large portion of $I$. If we change the word "bigger" to "smaller", we get the class of $\left(A_{\infty}\right)$ functions (see various definitions of $\left(A_{\infty}\right)$ on pp. 196-197, and 218 of [St]). In the classical case (see [St], pp. 200-205) the class $\left(A_{\infty}\right)$ comes into play as follows. The singular integral operator is "factorized" through the maximal operator $M$ in the sense that the weighted estimate $\|H f\|_{W} \leq C\|f\|_{W}$ splits into estimates $\|H f\|_{W} \leq C\|M f\|_{W}$ and $\|M f\|_{W} \leq C\|f\|_{W}$.

The proof of the first estimate uses the good $\lambda$ - inequality, which, roughly speaking, amounts to the fact that $|H| \leq C M$ up to a "telescopically graded" set. More precisely, $W(\{|H f|>2 \lambda\} \backslash\{M f<\delta \lambda\}) \leq \frac{1}{2} W(\{|H f|>\lambda\})$ for any $\lambda>0$ if $\delta>0$ is small. This property follows from the $\left(A_{\infty}\right)$ property of $W$.

To some extent, but in a more mysterious and strange way, the same two ideas (splitting the problem into two inequalities, where one of them amounts to the $\left(A_{\infty}\right)$ property of weight and uses the good $\lambda$-inequality) work in this paper.

But we will really factorize our operator, $H=E_{U}^{*} \cdot D \cdot E_{W}$, where $U=W^{-q / p}, q=$ $p /(p-1)$, and where $E$ is an embedding operator. It will embed to a (defined below) Triebel-Lizorkin space $f_{p}^{02}\left(\mathbb{C}^{d}\right)$. $D$ is an almost diagonal operator in the sequence space $f_{p}^{02}\left(\mathbb{C}^{d}\right)$. Those readers who preferred to read the main body of 
this article before reading the introduction may recognize the geometric meaning of $U: U^{1 / q}$ defines the metric which is conjugate to the metric defined by $W^{1 / p}$. In this factorization, the $\left(A_{\infty}\right)$ property of the weight $W$ will be used to prove the boundedness of the first factor $E_{W}$ (and not of the second "factor" as it happened in the classical case treated on pp. 201-207 of [St]). Actually the property of the matrix weight $W$ corresponding to $\left(A_{\infty}\right)$ will be called $\left(A_{p, \infty}\right)$. For matrix weights there is a whole spectrum of $\left(A_{p, \infty}\right)$ classes graded by $p \in(1, \infty)$ - they all coincide with the usual $\left(A_{\infty}\right)$ in the scalar situation.

So, as we will see, the boundedness of $H$ follows from $W \in\left(A_{p, \infty}\right), U \in\left(A_{q, \infty}\right)$. Together, these two properties of $W$ are equivalent to the matrix $\left(A_{p}\right)$ condition imposed on $W$.

In proving this equivalence we come across a Carleson measure characterization of (scalar) $\left(A_{\infty}\right)$ weights obtained in [FKP], [Bu1], and [Bu2]. For example, the matrix $\left(A_{2, \infty}\right)$ condition is equivalent to the requirement that the following measure in the disc is a Carleson measure:

$$
\mu \stackrel{\text { def }}{=} \sum_{I \in \mathcal{D}}\left\|\langle W\rangle_{I}^{-1 / 2}\left(\langle W\rangle_{I_{-}}-\langle W\rangle_{I_{+}}\right)\langle W\rangle_{I}^{-1 / 2}\right\| \cdot|I| \cdot \delta_{c_{I}}
$$

Here $c_{I}$ denotes the center of the Carleson box built on the arc $I: Q_{I} \stackrel{\text { def }}{=}\{z \in$ $\mathbb{D}: 1-|I| \leq|z|<1, z /|z| \in I\}$. This idea from [FKP], [Bu1], [Bu2] that $\left(A_{\infty}\right)$ is equivalent to a certain Carleson measure condition for the measures built by the averages of $W$, turns out to be extremely useful in [TV1], [TV2], [TV3] for proving that $E_{W}$ and $E_{U}$ are the correct embeddings. This is not surprising because Carleson measure is what embeddings are about.

Even though we do not know such a neat characterization of $\left(A_{p, \infty}\right)$ if $p$ is not 2 , we managed to prove that $\left(A_{p, \infty}\right)$ is equivalent to the embedding we need. This is done in Section 3, after explaining the matrix $\left(A_{p}\right)$ condition in Section 1, and the matrix $\left(A_{p, \infty}\right)$ condition in Section 2. Then we prove the boundedness of the Hilbert transform $H$ in Sections 4 and 5. To do this we will need the second ingredient from the classical theory: we use a certain variant of the good $\lambda$-inequality, the idea of which one can trace back to the works of Burkholder and Gundy $[\mathrm{BG}]$ and Axler, Chang, and Sarason $[\mathrm{AChS}]$.

The latter work is especially important because of its operator theory motivation.

Operator theory motivation. There is a part of the theory of singular integrals which treats the Hardy spaces in $\mathbb{R}^{n}$. The passage from $\mathbb{R}^{1}$ (or $\mathbb{T}$ ) to $\mathbb{R}^{n}$ makes the theory immensely richer. At the same time the theory of vector-valued Hardy spaces on $\mathbb{T}$ was developing for the needs of the spectral theory of operators (see [Nik]), because the dilation theory of linear contractions (see [Nik]) reduces questions about bounded operators in Hilbert space to functon theoretic questions in a vector Hardy space. Even a finite-dimensional case is known to be much richer than the scalar case (see [Nik] again). So the increase in dimension in this direction also enriches the theory. The connection with singular integrals becomes manifest if one considers Hankel and Toeplitz operators, given by formulae $H_{F} f \stackrel{\text { def }}{=} P_{-}(F f)=\left(I-P_{+}\right)(F f)$ and $T_{F} f \stackrel{\text { def }}{=} P_{+}(F f)$, where $F$ is a $d \times d$ matrix function and $f \in H^{p}\left(\mathbb{C}^{d}\right)$. One such problem which is very difficult already in the scalar case $d=1$ was considered in [AChS], and then a similar problem was considered in [S1], [S2]. It is closely related to a two-weight estimate for the Hilbert transform which is still open (see e.g. [TVZ], and the literature cited there). 
There is another classical problem for Toeplitz operators which leads to a weighted estimate (with one weight) for the Hilbert transform. In fact, the invertibility of the Toeplitz operator $T_{F}$ on $H^{p}$ is equivalent (see [Si]) to the factorization $F=G_{1}^{*} \cdot G_{2}$, where $G_{1}, G_{2}$ are $d \times d$ outer matrix functions such that the following estimate holds:

$$
\int_{\mathbb{T}}\left(V(t) P_{+} f(t), P_{+} f(t)\right) d m(t) \leq C \int_{\mathbb{T}}(W(t) f(t), f(t)) d m(t),
$$

where we denote $W=G_{1} G_{1}^{*}, V=\left(G_{2}^{-1}\right)^{*} G_{2}^{-1}$.

On first glance the matrix weights $W=G_{1} G_{1}^{*}, V=\left(G_{2}^{-1}\right)^{*} G_{2}^{-1}$ seem to be different, but the invertibility of $T_{F}$ implies easily $F^{-1} \in L_{d \times d}^{\infty}$, which means that the matrix weights $V, W$ are equivalent in the sense that there exists a constant $C$ such that for all $e \in \mathbb{C}^{d}$ and for almost all $t \in \mathbb{T}$

$$
\frac{1}{C}(V(t) e, e) \leq(W(t) e, e) \leq C(V(t) e, e) .
$$

This is how one can come to the matrix weighted norm inequality considered in this paper.

As far as we know, the first results about the matrix weight inequality were obtained by Steven Blum [Bl1], [Bl2], who noticed that if the matrix weight $W$ is assumed to be appropriately "smooth", then it can be diagonalized by a "smooth" unitary matrix function; and the operator of multiplication on this unitary matrix function commutes with $P_{+}$up to a compact term (because of "smoothness"). This approach leads to the pointwise diagonalization of the estimate under consideration and so to the corresponding scalar problem.

In the present work the matrix function $W$ is a priori arbitrary. Rather than doing pointwise diagonalization (which is not available now) we prefer to come to global (almost) diagonalization of our operator in the weighted space $L^{p}(W)$.

Notation. The symbol $f_{p}^{02}$ denotes the sequences $s=\left\{s_{I}\right\}_{I \in \mathcal{D}}$ enumerated by the set of dyadic intervals $\mathcal{D}$ such that

$$
\|s\|_{f_{p}^{02}}^{p}=\int\left(\sum_{I \ni x}\left|s_{I}\right|^{2} \frac{1}{|I|}\right)^{p / 2} d x<\infty .
$$

Similarly one can introduce $f_{p}^{02}\left(\mathbb{C}^{d}\right)$ by just replacing numbers $s_{I}$ by $\mathbb{C}^{d}$ vectors $s_{I}$ and $|\cdot|$ by $\|\cdot\|$. One can consider $f_{p}^{02}(w)$, which is a weighted Triebel-Lizorkin space. This amounts to changing the integration $d x$ to $w(x) d x$. As to the vector weighted norm one just replaces $\left\|s_{I}\right\|^{2}$ by $\left\|W^{1 / p}(x) s_{I}\right\|^{2}$ to obtain the definition of $f_{p}^{02}\left(\mathbb{C}^{d}, W\right)$, where $W(x)$ is now a positive selfadjoint operator (matrix).

\section{Matrix $A_{p}$ CONDition}

Let \|\| denote the standard Hilbert norm in $\mathbb{C}^{d}$, and let $($,$) be the scalar$ product in $\mathbb{C}^{d}$. If $\rho$ denotes a norm in $\mathbb{C}^{d}$, we can notice that $\rho(x) \asymp\|A x\|$, where $A$ is a positive selfadjoint operator and constants depend only on the dimension $d$. In fact, let us consider the ellipsoid of the largest volume contained in the $\rho$-unit ball $\{x: \rho(x) \leq 1\}$, which is a convex subset of $\mathbb{C}^{d}$. Clearly, $\{x:\|A x\| \leq 1\} \subset\{x$ : $\rho(x) \leq 1\} \subset C_{d}\{x:\|A x\| \leq 1\}$, where $A$ is a positive matrix mapping the ellipsoid onto the standard Hilbert ball. 
If we have a family $\rho_{t}$ of norms and consider $L^{p}(\rho)=\left\{f:\left(\int \rho_{t}(f)^{p} d t\right)^{1 / p}<\infty\right\}$, then we can also introduce an equivalent norm $\|f\|_{L^{p}(\rho)} \asymp\left(\int\|A(t) f\|^{p} d t\right)^{1 / p}$, with $A(t)$ as above constructed for $\rho_{t}$.

In weighted matrix inequalities one deals usually with $L^{p}(W)$ spaces, where $W(t)$ is matrix function with positive (a.e.) $W(t): \mathbb{C}^{d} \rightarrow \mathbb{C}^{d}$, and $\|f\|_{L^{p}(W)} \stackrel{\text { def }}{=}$ $\left(\int\left\|W(t)^{1 / p} f\right\|^{p}\right)^{1 / p}$.

As we see one can consider $L^{p}(\rho)$ as well and reduce it to $L^{p}(W)$ by setting $A(t)=W^{1 / p}(t)$.

Sometimes it is more convenient to use the language of $\rho$ and sometimes that of $W$.

Recall that the dual metric $\rho^{*}$ is given by

$$
\rho^{*}(x)=\sup _{y \neq 0} \frac{|(x, y)|}{\rho(y)} .
$$

We can identify $\left(L^{p}(\rho)\right)^{*}$ with $L^{q}\left(\rho^{*}\right), \frac{1}{p}+\frac{1}{q}=1$, and $\left(L^{p}(W)\right)^{*}$ with $L^{q}(U)$, where the $W^{1 / p}$-metric should be dual to the $U^{1 / q}$-metric, that is,

$$
W^{1 / p}(t)=U^{-1 / q}(t), \quad \text { for a.e. } t .
$$

To introduce the analog of $A_{p}$ for matrix weights we use the language of metrics $\rho$ and their averagings. Consider the norms ( $I$ is any interval)

$$
\rho_{p, I}(x) \stackrel{\text { def }}{=}\left(\frac{1}{I} \int_{I} \rho_{t}^{p}(x) d t\right)^{1 / p}
$$

The unit ball again can be "reduced" to an ellipsoid, that is, there exists a positive $A_{I}$ such that

$$
\rho_{p, I}(x) \underset{d}{\asymp}\left\|A_{I} x\right\| .
$$

Let us reduce the dual metric as well:

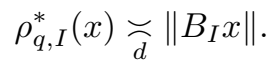

Proposition 1.1. 1) $|(x, y)| \leq \rho_{p, I}(x) \rho_{q, I}^{*}(y)$.

2) $\left(\rho_{p, I}\right)^{*} \leq \rho_{q, I}^{*}$.

Proof. 1) Obvious:

$$
|(x, y)|=\frac{1}{I} \int_{I}|(x, y)| \leq \frac{1}{I} \int_{I} \rho_{t}(x) \rho_{t}^{*}(y) \leq \rho_{p, I}(x) \rho_{q, I}^{*}(y) .
$$

2) This is a paraphrase of the first assertion.

Definition. If we have an opposite inequality

$$
\rho_{q, I}^{*} \leq C\left(\rho_{p, I}\right)^{*}, \quad \forall I,
$$

then $\rho$ is called an $A_{p}$-metric. If $\rho_{t}(x)=\left\|W^{1 / p}(t) x\right\|$ and $\rho$ is an $A_{p}$-metric, then $W$ is called an $A_{p}$ (matrix) weight.

Proposition 1.2. The following assertions are equivalent:

1) $\rho$ is an $A_{p}$-metric;

2) $\rho_{p, I} \leq C\left(\rho_{q, I}^{*}\right)^{*}$; 
3) if $\rho_{p, I}(x) \asymp\left\|A_{I} x\right\|, \rho_{q, I}^{*}(x) \asymp\left\|B_{I} x\right\|$, then $B_{I}^{2} \asymp A_{I}^{-2}$ in the sense of selfadjoint operators.

Proof. Obvious.

Remark. For $p=q=2$ the operators $A_{I}$ and $B_{I}$ can be calculated if we use the language of matrix weight $W$,

$$
\begin{gathered}
A_{I}=\langle W\rangle_{I}^{1 / 2}, B_{I}=\left\langle W^{-1}\right\rangle_{I}^{1 / 2} . \\
\langle W\rangle_{I}^{-1} \leq\left\langle W^{-1}\right\rangle_{I} .
\end{gathered}
$$

The $A_{2}$-condition obtained in [TV1], [TV2] had a form

$$
\left\langle W^{-1}\right\rangle_{I} \leq C\langle W\rangle_{I}^{-1},
$$

where inequality is understood in the sense of selfadjoint operators.

\section{Matrix $A_{\infty}$ CONDition}

Let us introduce the $A_{p, \infty}$ condition on metric weight which serves as the analog of the $A_{\infty}$ condition for scalar weights. Its definition goes exactly along the lines of the definition of $A_{p}$, except $\rho_{q, I}^{*}$ is replaced by

$$
\rho_{o, I}^{*}(x) \stackrel{\text { def }}{=} e^{\frac{1}{I} \int_{I} \log \rho_{t}^{*}(x) d t} .
$$

Proposition 2.1. $\left(\rho_{p, I}\right)^{*} \leq \rho_{o, I}^{*}$.

Proof. The inequality can be rewritten as

$$
\left(\rho_{o, I}^{*}\right)^{*} \leq \rho_{p, I} .
$$

Then it follows from two elementary observations:

$$
\rho_{o, I} \leq \rho_{p, I},
$$

which is just Jensen's inequality, and

$$
\left(\rho_{o, I}^{*}\right)^{*} \leq \rho_{o, I} .
$$

This last inequality follows from

$$
\log |(x, y)|-\frac{1}{I} \int_{I} \log \rho_{t}^{*}(y) d t \leq \frac{1}{I} \int_{I} \log \rho_{t}(x) d t,
$$

and this is the integration of the logarithm of $|(x, y)| \leq \rho_{t}^{*}(y) \rho_{t}(x)$.

Definition. If we have an opposite inequality

$$
\rho_{o, I}^{*} \leq C\left(\rho_{p, I}\right)^{*}, \quad \forall I
$$

which is equivalent to

$$
\rho_{p, I} \leq C\left(\rho_{o, I}^{*}\right)^{*}, \quad \forall I,
$$

then $\rho$ is called an $A_{p, \infty}$-metric. If $\rho_{t}(x)=\left\|W^{1 / p}(t) x\right\|$ and $\rho$ is an $A_{p, \infty}$-metric, then $W$ is called an $A_{p, \infty}$ matrix weight.

Remark. By Jensen's inequality $\rho_{o, I}^{*} \leq \rho_{q, I}^{*}$ and thus $A_{p} \Rightarrow A_{p, \infty}$.

Let $B_{\rho}$ denote the unit ball in the metric $\rho$.

Proposition 2.2. The following assertions are equivalent:

1) $\rho \in A_{p, \infty}$; 
2) $\frac{1}{I} \int_{I} \log \operatorname{vol} B_{\rho_{t}} d t \leq C+\log \operatorname{vol} B_{\rho_{p, I}}$ for all $I$.

In particular, $W \in A_{p, \infty}$ if and only if

$$
\operatorname{det} A_{I} \leq C e^{\frac{1}{I} \int_{I} \log \operatorname{det} W(t)^{1 / p} d t}, \quad \forall I,
$$

where $A_{I}$ is a positive definite operator reducing $\rho_{p, I}$ in the previous sense, namely

$$
\rho_{p, I}(x) \asymp\left\|A_{I} x\right\| .
$$

Proof. 1) $\Rightarrow 2$ ). It is convenient to use the language of matrices. So let $\rho_{t}(x) \asymp$ $\|A(t) x\|$ and $\rho_{p, I}(x) \asymp\left\|A_{I} x\right\|$. Let $\left\{e_{i}\right\}_{i=1}^{d}$ be an orthonormal basis of eigenvectors of $A_{I}$. We apply 1 ) to each of these vectors:

$$
\frac{1}{I} \int_{I} \log \left\|A(t)^{-1} e_{i}\right\| \leq C+\log \left\|A_{I}^{-1} e_{i}\right\|, \quad i=1, \ldots, d .
$$

Use the following elementary observation: for any positive definite $d \times d$ matrix $Q$ and any orthonormal basis $\left\{e_{i}\right\}_{i=1}^{d}$ in $\mathbb{C}^{d}$, $\operatorname{det} Q \leq \prod_{i=1}^{d}\left(Q e_{i}, e_{i}\right) \leq \prod_{i=1}^{d}\left\|Q e_{i}\right\|$. This inequality is just the consequence of arithmetic mean-geometric mean inequality. Summing up the inequalities above we get

$$
\frac{1}{I} \int_{I} \log \operatorname{det} A(t)^{-1} d t \leq C+\log \operatorname{det} A_{I}^{-1},
$$

which means

$$
\frac{1}{I} \int_{I} \log \operatorname{vol} B_{\rho_{t}} \leq C+\log \operatorname{vol} B_{\rho_{p, I}} .
$$

$2) \Rightarrow 1)$. Let $g$ be a unit vector. Choose $g_{1}(t) \equiv g, g_{2}(t), \ldots, g_{d}(t)$ to be unit vectors such that for $C(t) \stackrel{\text { def }}{=} A_{I}^{-1} A(t)$ we have $\operatorname{det} C(t)^{-1} \geq C_{d} \prod_{i=1}^{d}\left\|C(t)^{-1} g_{i}(t)\right\|$. We know that

$$
\begin{aligned}
\frac{1}{I} \int_{I}\left\|C(t) g_{i}(t)\right\|^{p} & \leq C_{d, p} \sum_{j=1}^{d} \frac{1}{I} \int_{I}\left|\left(C^{*}(t) e_{j}, g_{j}(t)\right)\right|^{p} \\
& \leq C_{d, p} \sum_{j=1}^{d}\left\|A(t) A_{I}^{-1} e_{j}\right\|^{p} \asymp C_{d, p} \sum_{j=1}^{d} \rho_{p, I}^{p}\left(A_{I}^{-1} e_{j}\right) \\
& <C_{d, p}^{\prime}<\infty .
\end{aligned}
$$

In particular,

$$
\sum_{i=1}^{d} \frac{1}{I} \int_{I} \log ^{+}\left\|C(t) g_{i}(t)\right\| d t \leq \frac{1}{p} C_{d, p}^{\prime} .
$$

We are given $\frac{1}{I} \int_{I} \log \operatorname{det} C(t)^{-1} d t \leq C$ which implies

$$
\begin{aligned}
& \frac{1}{I} \int_{I} \log ^{+}\left\|C(t)^{-1} g\right\| d t \leq C+\sum_{i=1}^{d} \frac{1}{I} \int_{I} \log ^{-}\left\|C(t)^{-1} g_{i}(t)\right\| \\
& \leq C+\sum_{i=1}^{d} \frac{1}{I} \int_{I} \log ^{+}\left\|C(t) g_{i}(t)\right\| d t \leq C+\frac{1}{p} C_{d, p}^{\prime}=C^{\prime \prime}<\infty .
\end{aligned}
$$


This implies

$$
\frac{1}{I} \int_{I} \log \left\|A(t)^{-1} g\right\| d t \leq C^{\prime \prime}+\log \left\|A_{I}^{-1} g\right\|
$$

The matrix $A_{2, \infty}$ condition can be reformulated (see [TV1]) in terms of the Carleson property of a certain measure built using $\left\|W_{I}^{-1 / 2}\left(W_{I_{1}}-W_{I_{2}}\right) W_{I}^{-1 / 2}\right\|$ exactly as it is done for scalar $A_{\infty}$ in Fefferman, Kenig and Pipher's work [FKP]. The same should probably be true for $A_{p, \infty}$ if some $A_{I}$-built measure is used.

\section{EMBEDding THEOREM}

In this section we deal with $\rho \in A_{p, \infty}$. We consider the "reducing" selfadjoint operators $A_{I}$ :

$$
\left(\frac{1}{I} \int_{I} \rho_{t}(x)^{p} d t\right)^{1 / p} \asymp\left\|A_{I} x\right\|
$$

Then the $A_{p, \infty}$ condition can be written as $\left(\rho_{p, I}\right)^{*} \geq c \rho_{o, I}^{*}$, that is,

$$
\forall J, \quad \frac{1}{J} \int_{J} \log \rho_{t}^{*}(x) d t \leq C+\log \left\|A_{J}^{-1} x\right\|,
$$

with the inverse inequality being always valid as well with $C=0$ (see Proposition 2.1).

Lemma 3.1. Let $\rho \in A_{p, \infty}$, and given a dyadic I let $\mathcal{F}_{k}$ denote the family of dyadic $J, J \subset I$, such that

$$
\left\|A_{J}^{-1} A_{I}\right\| \geq e^{k}
$$

Then

$$
\left|\bigcup_{J \in \mathcal{F}_{k}} J\right| \leq \frac{c}{k}|I| .
$$

Proof. Let $\left\{e_{i}\right\}_{i=1}^{d}$ be a standard basis in $\mathbb{C}^{d}$. Denote by $\mathcal{F}_{k}^{i}$ the set of dyadic intervals with

$$
\left\|A_{J}^{-1} A_{I} e_{i}\right\| \geq e^{k} .
$$

First use (3.2) to conclude that

$$
\frac{1}{I} \int_{I} \log \rho_{t}^{*}\left(A_{I} e_{i}\right) \leq C
$$

Then use the inverse to (3.2) to conclude that for $J \in \mathcal{F}_{k}^{i}$

$$
\frac{1}{J} \int_{J} \log ^{+} \rho_{t}^{*}\left(A_{I}, e_{i}\right) \geq \log \left\|A_{J}^{-1} A_{I} e_{i}\right\| \geq k .
$$


If we could replace log by $\log ^{+}$in (3.4) we would be done by standard argument. To do this replacement let us notice that

$$
\begin{aligned}
& \frac{1}{I} \int_{I} \log ^{+} \rho_{t}^{*}\left(A_{I} e_{i}\right)=\frac{1}{I} \int_{I} \log \rho_{t}^{*}\left(A_{I} e_{i}\right)+\frac{1}{I} \int_{I} \log ^{+} \frac{1}{\rho_{t}^{*}\left(A_{I} e_{i}\right)} \\
& \leq C+\frac{1}{p I} \int_{I} \log ^{+} \frac{1}{\left(\rho_{t}^{*}\left(A_{I} e_{i}\right)\right)^{p}} \leq C+\frac{1}{p I} \int_{I} \log ^{+}\left(\rho_{t}\left(A_{I}^{-1} e_{i}\right)\right)^{p} \\
& \quad \leq C+\frac{1}{p I} \int_{I} \rho_{t}^{p}\left(A_{I}^{-1} e_{i}\right) \leq C+\frac{1}{p}\left(C\left\|e_{i}\right\|\right)^{p} \leq C^{\prime} .
\end{aligned}
$$

In this chain we used an elementary inequality

$$
\rho_{t}\left(A_{I}^{-1} e_{i}\right) \rho_{t}^{*}\left(A_{I} e_{i}\right) \geq\left|\left(e_{i}, e_{i}\right)\right|=1 .
$$

The lemma is proved.

Remark. It is needless to say that for the scalar situation all $A_{p, \infty}$ just coincide with $A_{\infty}$. Lemma 3.1 reflects a characteristic property of $A_{\infty}$ functions: the set where the function is much smaller than its average is small.

Let $h_{J}$ denote a standard Haar function normalized in $L^{2}(d t)$.

Before stating the theorem we need to introduce a new type of weighted TriebelLizorkin spaces. Given a family of linear operators $\left\{C_{J}\right\}_{J \in D}$ we consider the space $f_{p}^{02}\left(\mathbb{C}^{d},\left\{C_{J}\right\}\right)$ to consist of sequences $\{s\}_{J \in D}$ such that $\left\{C_{J} s_{J}\right\} \in f_{p}^{02}$.

The following is the embedding theorem which gives the name to the section. As always $\frac{1}{p}+\frac{1}{q}=1$.

Theorem 3.2. Let $\rho \in A_{p, \infty}$ and let $\left\{A_{J}\right\}$ be its reducing operators as in (3.1). Let $s_{J}=\int_{J} g h_{J}$. Then the following inequality holds:

$$
\left\|\left\{s_{J}\right\}\right\|_{f_{q}^{02}\left(\left\{A_{J}^{-1}\right\}\right)} \leq C\|g\|_{L^{q}\left(\rho^{*}\right)} .
$$

Remarks. 1) Even in the simplest case $p=q=2$ and the scalar weight $w \in A_{\infty}$ this result seems to be rather new (although we did not check all the literature). It amounts to the inequality

$$
w \in A_{\infty} \Rightarrow \sum_{J \in D}\langle w\rangle_{J}^{-1}\left|\left(g, h_{J}\right)\right|^{2} \leq C\|g\|_{L^{2}\left(\frac{1}{w}\right)}^{2},
$$

which was mentioned in [TV1], [TVZ]. Notice that when $w \in A_{2}$ the left part is equivalent to

$$
\begin{aligned}
\sum\left\langle w^{-1}\right\rangle_{J}\left|\left(g, h_{J}\right)\right|^{2} & =\int \sum_{J \ni x}\left|\left(g, h_{J}\right)\right|^{2} \frac{1}{|J|} w^{-1}(x) d x \\
& =\left\|\left\{\left(g, h_{J}\right)\right\}\right\|_{f_{2}^{02}\left(w^{-1}\right)}^{2} \asymp\|g\|_{L^{2}\left(w^{-1}\right)}^{2} .
\end{aligned}
$$

2) There are interesting general relationships between

$$
f_{p}^{02}\left(\mathbb{C}^{d}, \rho\right) \stackrel{\text { def }}{=}\left\{\left\{s_{I}\right\}: \int\left(\sum_{I \ni t} \rho_{t}^{2}\left(s_{I}\right) \frac{1}{|I|}\right)^{p / 2} d t<\infty\right\}
$$


and

$$
f_{p}^{02}\left(\mathbb{C}^{d},\left\{C_{I}\right\}\right)=\left\{\left\{s_{I}\right\}: \int\left(\sum_{I \ni t}\left\|C_{I} s_{I}\right\|^{2} \frac{1}{|I|}\right)^{p / 2} d t<\infty\right\} .
$$

Quite naturally one should choose $C_{I}$ reducing $\rho_{t}$ :

$$
\left(\frac{1}{I} \int_{I} \rho_{t}^{p}(x) d t\right)^{1 / p} \asymp\left\|C_{I} x\right\|
$$

and then

$$
f_{p}^{02}\left(\mathbb{C}^{d},\left\{C_{I}\right\}\right)=\left\{\left\{s_{I}\right\}: \int\left(\sum_{I \ni t}\left(\frac{1}{I} \int_{I} \rho_{s}^{p}\left(s_{I}\right) d s\right)^{2 / p} \frac{1}{|I|}\right)^{p / 2} d t<\infty\right\} .
$$

It is clear that for $p=2$ these spaces coincide. It will be proved elsewhere that for $p \geq 2, \rho \in A_{p, \infty}$ they also coincide. Clearly, many questions appear: what about $p<2$ ?, what if we change exponent $p$ to another one in (3.6)?, etc.

Notice that if $W \in A_{p}$ these spaces coincide.

3) The space $f_{q}^{02}\left(\mathbb{C}^{d},\left\{A_{J}^{-1}\right\}\right)$ is not of the type considered in 3$)$. The difference is that now $C_{J}=A_{J}^{-1}$ are not generated as reducing operators as it was in (3.6). They are inverses to reducing operators.

Notice that if $W \in A_{p}$, then inverses to reducing operators are also reducing for the dual weight $U=W^{-q / p}$ and in this particular case we are going to prove in the next section that

$$
L^{p}(W) \approx f_{p}^{02}\left(\mathbb{C}^{d},\left\{A_{J}\right\}\right)
$$

where $A_{J}$ are reducing operators introduced in (3.1).

Proof of Theorem 3.2. Fix $g \in L^{q}\left(\rho^{*}\right)$ and a test function $f \in L^{p}$. We need to show that

$$
\sum_{J \in D}\left|\left(\int g h_{J}, A_{J}^{-1} \int f h_{J}\right)\right| \leq C\|f\|_{L^{p}}\|g\|_{L^{q}\left(\rho^{*}\right)}
$$

The sum on the left can be rewritten as

$$
\int\left(\sum_{J \ni t}\left|\left(\int g h_{J}, A_{J}^{-1} \int f h_{J}\right)\right| \frac{1}{|J|}\right) d t \stackrel{\text { def }}{=} \int S(t) d t .
$$

Let us consider a nonnegative function $h(t)$ and introduce

$$
S_{h(t)}(t)=\sum_{\substack{J \ni t \\|J| \leq h(t)}}\left|\left(\int g h_{J}, A_{J}^{-1} \int f h_{J}\right)\right| \frac{1}{|J|} .
$$

$S_{h(t)}(t) \leq S(t)$ but

$$
\int S_{h(t)}(t) d t \geq a \int S(t) d t
$$

if the function $h(t)$ has the following property:

$$
\forall J \in D \quad|\{t \in J: h(t) \geq|J|\}| \geq a|J| .
$$

We are going to choose now a function $h$ satisfying (3.9) and such that

$$
S_{h(t)}(t) \leq B\left(M\|f\|^{p_{*}}\right)^{1 / p_{*}}(t)\left(M\left(\rho^{*}(g)\right)^{q_{*}}\right)^{1 / q_{*}}(t)
$$


for certain $B<\infty, 1<p_{*}<p, 1<q_{*}<q$. $M$ denotes the maximal function. After (3.10) we are done:

$$
\begin{aligned}
\int S(t) d t & \leq a^{-1} \int S_{h(t)}(t) d t \leq a^{-1} \int\left(M\|f\|^{p_{*}}\right)^{1 / p_{*}}(t) \cdot\left(M \rho^{*}(g)^{q_{*}}\right)^{1 / q_{*}}(t) \\
& \leq a^{-1}\left(\int\left(M\|f\|^{p_{*}}\right)^{p / p_{*}}\right)^{1 / p}\left(\int\left(M \rho^{*}(g)^{q_{*}}\right)^{q / q_{*}}\right)^{1 / q} \\
& \leq C\left(a, p / p_{*}, q / q_{*}\right)\left(\int\|f\|^{p}\right)^{1 / p}\left(\int \rho^{*}(g)^{q}\right)^{1 / q} \\
& =C\left(a, p / p_{*}, q / q_{*}\right)\|f\|_{L^{p}} \cdot\|g\|_{L^{q}\left(\rho^{*}\right)}
\end{aligned}
$$

which is what we need.

To choose $h(t)$ satisfying (3.9) and (3.10) we follow the algorithm below.

Fix a dyadic interval $I$. Fix a large $k$ and delete from $I$ all the dyadic intervals for which

$$
\left\|A_{I} A_{J}^{-1}\right\| \geq e^{k} .
$$

By Lemma 3.1 the set $E_{I}$ which rests has the property that

$$
\left|E_{I}\right| \geq \frac{1}{2}|I|
$$

Let us introduce

$$
\begin{aligned}
& t \in I, \quad S^{A_{I}^{-1}}(g)(t)=\left(\sum_{\substack{J \ni t \\
J \subseteq I}}\left\|A_{I}^{-1} \int g h_{J}\right\|^{2} \frac{1}{|J|}\right)^{1 / 2} ; \\
& t \in I, \quad S^{A_{I} A_{J}^{-1}}(f)(t)=\left(\sum_{\substack{J \ni t, J \subseteq I}}\left\|A_{I} A_{J}^{-1} \int f h_{J}\right\|^{2} \frac{1}{|J|}\right)^{1 / 2} .
\end{aligned}
$$

We are interested in proving that there are relatively large subsets of $I$ on which these $S$-functions are smaller than maximal functions from (3.10).

To find such sets it is sufficient to estimate any averages of these $S$-functions. Fix $\alpha>1$ and very close to 1 . Then

$$
\begin{aligned}
\frac{1}{I} \int_{I}\left(S^{A_{I}^{-1}}(g)(t)\right)^{\alpha} d t & \leq C(\alpha) \frac{1}{I} \int\left\|A_{I}^{-1} g \cdot \chi_{I}\right\|^{\alpha} d t \\
& =C(\alpha) \frac{1}{I} \int_{I}\left\|A_{I}^{-1} g\right\|^{\alpha} d t
\end{aligned}
$$

The first inequality follows from the classical $L^{\alpha}$ estimate of the $S$-function by the $L^{\alpha}$ norm of the function when $\alpha>1$ (see e.g. [St]). To continue the estimate we use that we are in the space of finite dimension to write $\left(\left\{e_{i}\right\}\right.$ is a standard basis of $\left.\mathbb{C}^{d}\right)$ :

$$
\left\|A_{I}^{-1} g(t)\right\| \leq C_{d} \sum_{i=1}^{d}\left|\left(A_{I}^{-1} g(t), e_{i}\right)\right| \leq C_{d} \sum_{i=1}^{d} \rho_{t}^{*}(g) \rho_{t}\left(A_{I}^{-1} e_{i}\right) .
$$


Thus

$$
\begin{aligned}
\left\|S^{A_{I}^{-1}}(g)\right\|_{L^{\alpha}\left(\frac{d t}{I}\right)} & \leq C(\alpha, d) \sum_{i=1}^{d}\left(\frac{1}{I} \int_{I} \rho_{t}^{(p+\varepsilon) \alpha}\left(A_{I}^{-1} e_{i}\right)\right)^{\frac{1}{(p+\varepsilon) \alpha}} \\
& \cdot\left(\frac{1}{I} \int_{I} \rho_{t}^{*}(g)^{(q-\delta) \alpha}\right)^{\frac{1}{(q-\delta) \alpha}}
\end{aligned}
$$

Here $(p+\varepsilon)^{-1}+(q-\delta)^{-1}=1$, and $\varepsilon$ is chosen to be very small to guarantee $q-\delta>1$. The choice of $\varepsilon$ and $\alpha$ is also dictated by the observation that the positive function $t \rightarrow \rho_{t}^{p}(x)$ is an $A_{\infty}$-function if $\rho \in A_{p, \infty}$.

In fact, inequalities (2.1) and (2.3) imply that

$$
\frac{1}{I} \int_{I} \rho_{t}^{p}(x) d t \leq C e^{\frac{1}{I} \int_{I} \log \rho_{t}^{p}(x) d t},
$$

which is equivalent to $A_{\infty}$ (see [GCRF] and [St], p. 218). As $\rho_{t}^{p}(x)$ is (uniformly in $x$ ) in $A_{\infty}$ it satisfies (uniformly in $x$ ) a reverse Hölder inequality. In particular, there exist a $\bar{p}>p$ such that

$$
\forall I, \forall x,\left(\frac{1}{I} \int_{I} \rho_{t}^{\bar{p}}(x) d t\right)^{1 / \bar{p}} \leq C\left(\frac{1}{I} \int_{I} \rho_{t}^{p}(x) d t\right)^{1 / p} .
$$

Now we choose $\alpha$ close to 1 and $\varepsilon$ close to 0 to guarantee that

$$
(p+\varepsilon) \alpha \leq \bar{p}
$$

In particular, (3.13) implies

$$
\begin{aligned}
\left(\frac{1}{I} \int_{I} \rho_{t}^{(p+\varepsilon) \alpha}\left(A_{I}^{-1} e_{i}\right) d t\right)^{\frac{1}{(p+\varepsilon) \alpha}} & \leq C\left(\frac{1}{I} \int_{I} \rho_{t}^{p}\left(A_{I}^{-1} e_{i}\right)\right)^{1 / p} \\
& \leq C^{\prime}\left\|A_{I} A_{I}^{-1} e_{i}\right\|=C^{\prime}
\end{aligned}
$$

where we use the definition (3.1) of $A_{I}$.

Now we can continue (3.11) using (3.14):

$$
\left\|S^{A_{I}^{-1}}(g)\right\|_{L^{\alpha}\left(I, \frac{d t}{T}\right)} \leq C(\alpha, d) \cdot d \cdot C^{\prime} \cdot \inf _{t \in I}\left[M\left(\rho^{*}(g)^{q_{*}}\right)(t)\right]^{\frac{1}{q_{*}}},
$$

where $q_{*}=(q-\delta) \cdot \alpha \in(1, q)$ if $\delta=\delta(\varepsilon)$ and $\alpha$ are chosen properly.

This last inequality ensures that for any $\tau, \tau \in(0,1)$, we can find the subset $E(\tau, I) \subset I,|E(\tau, I)| \geq\left(1-\tau^{\alpha}\right)|I|$ such that

$$
t \in E(\tau, I) \Rightarrow S^{A_{I}^{-1}}(g)(t) \leq \frac{C^{\prime \prime}(\alpha, d)}{\tau} \inf _{t \in I}\left[M\left(\rho^{*}(g)^{q_{*}}\right)\right]^{1 / q_{*}}
$$

This is the main estimate of our first $S$-function.

Now we will give a similar estimate for the second $S$-function $S^{A_{I} A_{J}^{-1}}(f)(t)$. Again choose $\alpha>1$ and close to 1 to estimate $\frac{1}{\left|E_{I}\right|} \int_{E_{I}}\left(S^{A_{I} A_{J}^{-1}}(f)(t)\right)^{\alpha} d t$. For $t \in E_{I}$ all $J \ni t, J \subset I$ have the property

$$
\left\|A_{I} A_{J}^{-1}\right\| \leq e^{k}
$$


and so

$$
t \in E_{I}, \quad S^{A_{I} A_{J}^{-1}}(f)(t) \leq S^{I}(f)(t) \stackrel{\text { def }}{=}\left(\sum_{\substack{J \ni t \\ J \subset I}}\left\|\int f h_{J}\right\|^{2} \frac{1}{|J|}\right)^{1 / 2}
$$

Thus

$$
\frac{1}{\left|E_{I}\right|} \int_{E_{I}}\left(S^{A_{I} A_{J}^{-1}}(f)(t)\right)^{\alpha} d t \leq \frac{2}{I} \int_{I}\left(S^{I}(f)(t)\right)^{\alpha} d t \leq C(\alpha) \frac{1}{I} \int_{I}\|f\|^{\alpha} d t .
$$

Choosing $1<\alpha<p$ and denoting it by $p_{*}$ we get

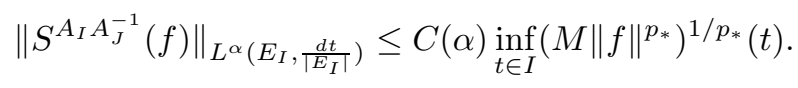

As before it implies for any $\tau \in(0,1)$ the existence of $e(\tau, I) \subset E_{I},|e(\tau, I)| \geq$ $\left(1-\tau^{\alpha}\right)\left|E_{I}\right| \geq \frac{1}{2}\left(1-\tau^{\alpha}\right)|I|$ such that

$$
t \in e(\tau, I) \Rightarrow S^{A_{I} A_{J}^{-1}}(f)(t) \leq \frac{C(\alpha)}{\tau} \inf _{t \in I}\left(M\|f\|^{p_{*}}\right)^{1 / p_{*}} .
$$

Now choose $\tau$ so small that

$$
|e(\tau, I) \cap E(\tau, I)| \geq \frac{1}{4}|I| .
$$

We are ready to choose the height function $h(t)$ satisfying (3.9) and (3.10). Put

$$
\begin{aligned}
h(t) \stackrel{\text { def }}{=} \sup \left\{h: S_{h}(t)\right. & \stackrel{\text { def }}{=} \sum_{\substack{J \ni t \\
|J| \leq h}} \frac{1}{|J|}\left|\left(\int g h_{J}, A_{J}^{-1} \int f h_{J}\right)\right| \\
& \leq B\left(M\|f\|^{p_{*}}\right)^{1 / p_{*}}(t)\left(M \rho^{*}(g)^{q_{*}}(t)\right\},
\end{aligned}
$$

where $p_{*}, q_{*}$ appeared in (3.17) and (3.16) and where $B=\frac{C^{\prime \prime}(\alpha, d) C(\alpha)}{\tau^{2}}$, with constants taken from (3.16) and (3.17).

Then (3.10) is fulfilled automatically. To check (3.9) let us fix any $I \in D$ and let us consider for $t \in I$

$$
S_{I}(t) \stackrel{\text { def }}{=} \sum_{\substack{J \ni t \\ J \subseteq I}} \frac{1}{|J|}\left|\left(\int g h_{J}, A_{J}^{-1} \int f h_{J}\right)\right| .
$$

Notice that

$$
S_{I}(t)=\sum_{\substack{J \ni t \\ J \subseteq I}}\left|\left(A_{I}^{-1} \int g h_{J}, A_{I} A_{J}^{-1} \int f h_{J}\right)\right| \leq S^{A_{I}^{-1}}(g)(t) S^{A_{I} A_{J}^{-1}}(f)(t) .
$$

Thus (3.16) and (3.17) applied for $t \in e(\tau, I) \cap E(\tau, I)$ show that on at least a quarter of $I$ (see (3.18))

$$
S_{I}(t) \leq B\left(M\|f\|^{p_{*}}\right)^{1 / p_{*}}(t)\left(M \rho^{*}(g)^{q_{*}}\right)^{1 / q_{*}}(t) .
$$

Thus on a quarter of $I$ the function $h$ just defined in (3.19) is greater than $|I|$. Because $I$ was arbitrary, (3.9) holds and Theorem 3.2 is finished. 


\section{4. $T_{ \pm}$THEOREM}

Theorem 3.2 means that the operator $\sum A_{J}^{-1} h_{J} \otimes h_{J}$ given by

$$
g \rightarrow \sum_{J \in D} A_{J}^{-1}\left(\int g h_{J}\right) \cdot h_{J}
$$

is bounded as $L^{q}\left(\rho^{*}\right) \rightarrow f_{q}^{02}$. If we use the language of matrix functions, meaning that $\rho_{t}(x)=\left\|W^{1 / p}(t) x\right\|, \rho_{t}^{*}(x)=\left\|U^{1 / q}(t) x\right\|$, where $U^{1 / q}=W^{-1 / p}$, we can make a change of variable $g(t)=W^{1 / p}(t) G(t)$ now having $G \in L^{q}$ with no weight. The operator now can be written in the form $\sum A_{J}^{-1} h_{J} \otimes W^{1 / p} h_{J}$. If $A_{J}$ is given by (3.1), the sum above is proved to be a bounded operator from $L^{q}$ to $f_{q}^{02}$. The same proof certainly shows that operators $\sum \pm A_{J}^{-1} h_{J} \otimes W^{1 / p} h_{J}$ are uniformly bounded.

All these claims are true under the condition that $\rho$ (or $W$ ) belongs to $A_{p, \infty}$.

Now if $\rho$ (or $W$ ) belongs to $A_{p}$ one can say more. We summarize it in two theorems.

Theorem 4.1. Let $\rho \in A_{p}$. Then if $A_{J}$ are from (3.1) and $s_{J}=\int f h_{J}$ we have

$$
\left\|\left\{s_{J}\right\}\right\|_{f_{p}^{02}\left(\left\{A_{J}\right\}\right)} \asymp\|f\|_{L^{p}(\rho)} .
$$

Proof. It follows immediately from the definition that $\rho \in A_{p} \Rightarrow \rho \in A_{p, \infty}$ and $\rho \in A_{p} \Rightarrow \rho^{*} \in A_{q} \Rightarrow \rho^{*} \in A_{q, \infty}$.

Let us also introduce the reducing operators for $\rho^{*}$ :

$$
\left(\frac{1}{J} \int_{J} \rho_{t}^{*}(x)^{q} d t\right)^{1 / q} \asymp\left\|C_{J} x\right\| .
$$

Let us apply Theorem 3.2 to $\rho^{*} \in A_{q, \infty}$ to obtain

$$
\left\|\left\{\int f h_{J}\right\}\right\|_{f_{p}^{02}\left(\left\{C_{J}^{-1}\right\}\right)} \leq C\|f\|_{L^{p}(\rho)} .
$$

Notice that $\rho \in A_{p}$ means that $C_{J}^{-2} \asymp A_{J}^{2}$ and so we get

$$
\left\|\left\{\int f h_{J}\right\}\right\|_{f_{p}^{02}\left(\left\{A_{J}\right\}\right)} \leq C\|f\|_{L^{p}(\rho)} .
$$

To obtain the inverse inequality we use duality and again Theorem 3.2 for $\rho \in A_{p, \infty}$ to obtain

$$
\begin{aligned}
\|f\|_{L^{p}(\rho)} & =\sup _{\|g\|_{L^{q}\left(\rho^{*}\right)} \leq 1}|\langle f, g\rangle|=\sup \left|\sum\left(\int f h_{J}, \int g h_{J}\right)\right| \\
& =\sup \left|\sum\left(A_{J} \int f h_{J}, A_{J}^{-1} \int g h_{J}\right)\right| \\
& \leq\left\|\left\{\int f h_{J}\right\}\right\|_{f_{p}^{02}\left(\left\{A_{J}\right\}\right)} \cdot\left\|\left\{\int g h_{J}\right\}\right\|_{f_{q}^{02}\left(\left\{A_{J}^{-1}\right\}\right)} \\
& \leq C\left\|\left\{\int f h_{J}\right\}\right\|_{f_{p}^{02}\left(\left\{A_{J}\right\}\right)} .
\end{aligned}
$$

The last inequality is Theorem 3.2. We are done. 
Remark. It seems to us that this theorem gives new information about $L^{p}(w), w \in$ $A_{p}$, even in the scalar case. The reader should notice that we organized a canonical isomorphism of $L^{p}(w)$ and $f_{p}^{02}$. This is not the classical isomorphism $f \rightarrow$ $\left\{\left(f, h_{J}\right)\right\}_{J \in D}$ between $L^{p}(w)$ and $f_{p}^{02}(w)$.

Corollary 4.2. Operators

$$
T_{ \pm} \stackrel{\text { def }}{=} \sum \pm h_{J} \otimes h_{J}
$$

defined by the formula

$$
T_{ \pm} \varphi=\sum_{J \in D} \pm h_{J} \cdot \int \varphi h_{J}
$$

are uniformly bounded in $L^{p}(\rho)$ if and only if $\rho \in A_{p}$.

Proof. The "if" part follows immediately from Theorem 4.1. In fact, we just use the equivalent norm $\|\cdot\|_{f_{p}^{02}\left(\left\{A_{J}\right\}\right)}$ in $L^{p}(\rho)$ which does not change if $\varphi$ is replaced by $T_{ \pm} \varphi$ because $\int T_{ \pm} \varphi \cdot h_{J}= \pm \int \varphi h_{J}$. The "only if" part follows from the uniform boundedness of rank one operators $h_{J} \otimes h_{J}$. It follows exactly as the first part of Theorem 5.1 below. So we send the reader to this coming proof.

The next result is an analog of the classical isomorphism $L^{p}(w) \approx f_{p}^{02}(w)$.

Theorem 4.3. Let $\rho \in A_{p}$. Let $s_{J}=\int f h_{J}$. Then

$$
\left\|\left\{s_{J}\right\}\right\|_{f_{p}^{02}(\rho)} \asymp\|f\|_{L^{p}(\rho)} .
$$

Proof. This follows easily from the corollary. In fact, we know that

$$
\|f\|_{L^{p}(\rho)}^{p} \asymp \int \rho_{t}^{p}\left(\sum_{J \in D} \pm h_{J} \cdot \int f h_{J}\right) d t .
$$

We integrate over the signs and use the Khintchin theorem which is valid here. We obtain

$$
\begin{aligned}
\|f\|_{L^{p}(\rho)}^{p} & \asymp \int\left(\sum_{J \ni t} \rho_{t}^{2}\left(\int f h_{J}\right) \frac{1}{|J|}\right)^{p / 2} d t \\
& =\left\|\left\{s_{J}\right\}\right\|_{f_{p}^{02}(\rho)}^{p} .
\end{aligned}
$$

Remark and question. In particular, if $\rho \in A_{p}$ and $A_{J}$ are reducing operators for $\rho$ considered in (3.1), we see from (4.1) and (4.2) the following equivalence of norms in sequence spaces:

$$
\left\|\left\{s_{J}\right\}\right\|_{f_{p}^{02}(\rho)} \asymp\left\|\left\{s_{J}\right\}\right\|_{f_{p}^{02}\left(\left\{A_{J}\right\}\right)} .
$$

But it has already been mentioned in the remarks to Theorem 3.2 that this equivalence does not require $\rho \in A_{p}$. For $p=2$ it holds always. For $p \geq 2, \rho \in A_{p, \infty}$ would be sufficient for (4.3). This can be proved along the lines of Theorem 3.2.

We would like to ask for the criterion for (4.3) to hold. 


\section{Hilbert transform}

Theorem 4.1 and Corollary 4.2 allow one to show that any Calderon Zygmund operator with $T 1=T^{*} 1=0$ is bounded in $L^{p}(\rho)$ if $\rho \in A_{p}$. But still the reduction of any $T$ to $T_{ \pm}$is a very technical and difficult task. In this section we show that at least for the Hilbert transform one can give a relatively easy and straightforward proof very similar to the one of Corollary 4.2.

Theorem 5.1. Let $\rho \in A_{p}$. Then

$$
\left\|P_{+} f\right\|_{L^{p}(\rho)} \leq C\|f\|_{L^{p}(\rho)} .
$$

Conversely if (5.1) holds, then $\rho \in A_{p}$.

Before proving Theorem 5.1 let us prove two simple lemmas. Consider the Poisson kernel $P_{I}=\frac{|I|}{x^{2}+|I|^{2}}$ and define $k_{I, q}=P_{I}^{q / 2} \cdot|I|^{q / 2-1} \quad(1<q<\infty)$. Then $\int k_{I, q} \asymp 1$ and here is the first lemma.

Lemma 5.2. If $u$ is a scalar $A_{q}$ weight, then $\int u k_{I, q} \leq C \frac{1}{|I|} \int_{I} u$.

Proof. We can write $k_{I, q} \asymp \sum_{k=1}^{\infty} \varepsilon_{k} \frac{1}{\left|2^{k} I\right|} \chi_{2^{k} I}$, where $\varepsilon_{k} \asymp\left(2^{-k}\right)^{q / p}$. Now if $u \in A_{q}$ let $w=u^{-p / q} \in A_{p}$ and we can write the following chain of inequalities:

$$
\begin{aligned}
\left(w_{I}\right)^{q / p}\left(\int k_{I, q} u\right) & \leq \sum_{k=1}^{\infty}\left(w_{I}\right)^{q / p} u_{2^{k} I} \varepsilon_{k} \\
& \leq \sum_{k=1}^{\infty} \frac{\left(w_{I}\right)^{q / p}}{\left(w_{2^{k} I}\right)^{q / p}} \varepsilon_{k}=\sum_{k=1}^{\infty}\left(\frac{w(I)}{w\left(2^{k} I\right)}\right)^{q / p} 2^{k \cdot \frac{q}{p}} \varepsilon_{k} \\
& \leq C \sum_{k=1}^{\infty}\left(\frac{w(I)}{w\left(2^{k} I\right)}\right)^{q / p} \leq C \sum_{k=1}^{\infty}(1+\varepsilon)^{-k} \leq C^{\prime} .
\end{aligned}
$$

The last inequality follows from the doubling property of $A_{p}$ weights. We get $\int k_{I, q} u \leq C\left(w_{I}\right)^{-q / p} \leq C^{\prime} u_{I}$ by using the $A_{p}$ condition again.

Lemma 5.3. If $W$ is a matrix $A_{p}$ weight, then $t \rightarrow\left\|W^{1 / p} x\right\|^{p}$ is a scalar $A_{p}$ weight for any vector $x$.

Proof. It is convenient to use the language of $\rho_{t}(x)=\left\|W^{1 / p}(t) x\right\|$. Fix $x$ and choose $y(x)$ in such a way that $\left(\rho_{p, I}\right)(x)=\sup _{y} \frac{|(x, y)|}{\left(\rho_{p, I}\right)^{*}(y)} \leq 2 \frac{\mid(x, y(x) \mid}{\left(\rho_{p, I}\right)^{*}(y(x))}$. Now

$$
\begin{aligned}
& \left(\frac{1}{|I|} \int_{I} \rho_{t}^{p}(x)\right)^{1 / p}\left(\frac{1}{I} \int_{I} \rho_{t}^{-q}(x)\right)^{1 / q} \leq \rho_{p, I}(x)\left(\frac{1}{I} \int_{I}\left(\frac{\rho_{t}^{*}(y(x))}{|(x, y(x))|}\right)^{q}\right)^{1 / q} \\
& \quad \leq \frac{\rho_{p, I}(x) \rho_{q, I}^{*}(y(x))}{|(x, y(x))|} \leq C \frac{\rho_{p, I}(x)\left(\rho_{p, I}\right)^{*}(y(x))}{|(x, y(x))|} \leq 2 C
\end{aligned}
$$

Proof of Theorem 5.1. (5.1) $\Rightarrow \rho \in A_{p}$. 
Operator $P_{+}$has the kernel $\frac{1}{1-\bar{\zeta} z}$. We are given that it is a bounded operator. Fix an $a$ in the unit disk and consider the operator with the kernel

$$
\frac{1}{1-\bar{\zeta} z}\left[1-\left(\frac{\overline{\zeta-a}}{1-\bar{a} \zeta}\right) \frac{z-a}{1-\bar{a} z}\right] \text {. }
$$

Obviously it is a bounded operator in $L^{p}(\rho)$ too, actually it is given by the formula $P_{+}-b_{a} P_{+} \bar{b}_{a}$, where $b_{a}(\zeta)=\frac{\zeta-a}{1-\bar{a} z}$. Notice that pointwise multiplication by $b_{a}$ does not change the norm in $L^{p}(\rho)$. Now let us make the simplification in the kernel to see that it equals $\frac{1-|a|^{2}}{(1-\bar{\zeta} a)(1-\bar{a} z)}=k_{a}(z) \otimes k_{a}(\zeta)$, where $k_{a}(\zeta)=\frac{\left(1-|a|^{2}\right)^{1 / 2}}{1-\bar{a} \zeta}$ is a normalized Szegö kernel in $L^{2}$. Notice that

$$
\left|k_{a}(\zeta)\right|^{2}=P_{a}(\zeta)
$$

where $P_{a}(\zeta)$ denotes the Poisson kernel.

Now let us use the fact (which we just derived from the boundedness of $P_{+}$) that $k_{a} \otimes k_{a}$ are uniformly bounded. Let $\varphi(t) \in L^{q}, \psi \in L^{p}$, and $\int\|\varphi\|^{q} d t \leq$ $1, \int\|\psi\|^{p} d t \leq 1$. Then

$$
\left|\left(\int k_{a}(t) W^{1 / p}(t) \varphi(t) d t, \int k_{a}(s) U^{1 / q}(s) \psi(s) d s\right)\right| \leq C
$$

uniformly in $a$. Denoting $k_{a}^{(p)}=\left(1-|a|^{2}\right)^{1 / 2-1 / p} k_{a}$ and $k_{a}^{(q)}=\left(1-|a|^{2}\right)^{1 / 2-1 / q} k_{a}$, we have

$$
\int\left|k_{a}^{(p)}(t)\right|^{p}\left\|W^{1 / p}(t) \int k_{a}^{(q)}(s) U^{1 / q}(s) \psi(s) d s\right\|^{p} \leq C .
$$

Denoting $z=\int k_{a}^{(q)}(s) U^{1 / q}(s) \psi(s) d s$ we conclude that

$$
\left(\int\left|k_{a}^{(p)}\right|^{p}\left\|W^{1 / p}(t) z\right\|^{p}\right)^{1 / p} \leq C\|\psi\|_{L^{p}}
$$

This is an averaging because $\int\left|k_{a}^{(p)}\right|^{p} \asymp 1$. Denote it by $\rho_{p, a}$ :

$$
\rho_{p, a}(z) \leq C\|\psi\|_{L^{p}}
$$

We want to show that

$$
\left(\rho_{q, a}^{*}\right)^{*}(z) \geq C \inf \left\{\|\psi\|_{L^{p}}: z=\int k_{a}^{(q)}(s) U^{1 / q}(s) \psi(s)\right\} .
$$

But

$$
\begin{aligned}
\left(\rho_{q, a}^{*}\right)^{*}(z) & =\max _{y}|(y, z)| /\left(\int\left\|U^{1 / q}(s) y\right\|^{q}\left|k_{a}^{(q)}(s)\right|^{q}\right)^{1 / q} \\
& =\max _{y}\left|\left\langle k_{a}^{(q)}(s) U^{1 / q}(s) y, \psi(s)\right\rangle\right| /\left\|k_{a}^{(q)}(s) U^{1 / q}(s) y\right\|_{L^{q}} \\
& =\inf \left\{\|\psi\|_{L^{p}}: z=\int k_{a}^{(q)}(s) U^{1 / q}(s) \psi(s) d s\right\}
\end{aligned}
$$

Thus

$$
\rho_{p, a}(z) \leq C\left(\rho_{q, a}^{*}\right)^{*}(z)
$$


But $\left|k_{a}^{(p)}\right|^{p} \geq C(p) \frac{\chi_{I_{a}}}{\left|I_{a}\right|}$, where $I_{a}$ is the interval of length $1-|a|$ centered at $a /|a|$. Thus

$$
\rho_{p, I} \leq C\left(\rho_{q, I}^{*}\right)^{*} .
$$

Now let us prove that $\rho \in A_{p} \Rightarrow(5.1)$.

Let $\rho$ be given by $W^{1 / p}$ and $\rho^{*}$ by $U^{1 / q}=W^{-1 / p}$. We have to prove that

$$
f \rightarrow W^{1 / p} P_{+} W^{-1 / p} f
$$

is a bounded operator as $L^{p} \rightarrow L^{p}$. Fix $g \in L^{q}$. We need to show

$$
\int\left|\left(\nabla P_{+} W^{-1 / p} f(z), \nabla P_{+} W^{1 / p} g(z)\right)\right| \delta(z) d A(z) \leq C\|f\|_{p}\|g\|_{q}
$$

where $\delta(z)$ means the distance to the boundary. In other words, introducing a Stolz cone $\Gamma_{t}$, and

$$
S(t) \stackrel{\text { def }}{=} \int_{\Gamma_{t}}\left|\left(\nabla P_{+} W^{-1 / p} f(z), \nabla P_{+} W^{1 / p} g(z)\right)\right| d A(z),
$$

one needs to prove that

$$
\int S(t) d t \leq C\|f\|_{p}\|g\|_{q}
$$

We follow closely the lines of the proof of Theorem 3.2. Let us consider a nonnegative function $h(t)$ and

$$
S_{h(t)}(t)=\int_{\Gamma_{t, h(t)}}\left|\left(\nabla P_{+} W^{-1 / p} f(z), \nabla P_{+} W^{1 / p} g(z)\right)\right| d A(z),
$$

where

$$
\Gamma_{t, h(t)}=\Gamma_{t} \cap\{z: \delta(z) \leq h(t)\} .
$$

As in Section 3

$$
\int S_{h(t)} d t \asymp \int S(t) d t
$$

if the function $h(t)$ has the following property:

$$
\forall J \quad|\{t \in J: h(t) \geq|J|\}| \geq a|J| .
$$

Let us choose $h$ to be maximal such that

$$
S_{h(t)}(t) \leq B\left(M\|f\|^{p_{*}}\right)^{1 / p_{*}}(t)\left(M\|g\|^{q_{*}}\right)^{1 / q_{*}}(t),
$$

where $B, p_{*} \in(1, p), q_{*} \in(1, q)$ will be chosen later.

If this $h$ satisfies (5.4), then (5.3) and (5.2) imply what we need.

To choose $B, p_{*}, q_{*}$ and to prove that $h$ satisfies (5.4) we follow the algorithm below.

Fix an interval $I$. Consider $f_{1}=f \cdot \chi_{2 I}, g_{1}=g \cdot \chi_{2 I}$ and $f_{2}=f-f_{1}, g_{2}=g-g_{1}$. Let us introduce $A_{I}$ as in (3.1):

$$
\begin{aligned}
& t \in I, \quad S^{A_{I}}\left(f_{i}\right)(t)=\left(\int_{\Gamma_{t,|I|}}\left\|\nabla P_{+} A_{I} W^{-1 / p} f_{i}\right\|^{2} d A(z)\right)^{1 / 2}, i=1,2 ; \\
& t \in I, \quad S^{A_{I}^{-1}}\left(g_{i}\right)(t)=\left(\int_{\Gamma_{t,|I|}}\left\|\nabla P_{+} A_{I}^{-1} W^{1 / p} g_{i}\right\|^{2} d A(z)\right)^{1 / 2}, i=1,2 .
\end{aligned}
$$


Then exactly as in Section 3 we choose $\alpha>1, \alpha$ close to 1 , and

$$
\begin{aligned}
\left\|S^{A_{I}}\left(f_{1}\right)\right\|_{L^{\alpha}\left(I, \frac{d t}{I}\right) \leq} \leq & C(\alpha, d)\left(\frac{1}{I} \int_{2 I}\|f\|^{(p-\delta) \alpha}\right)^{\frac{1}{(p-\delta) \alpha}} \\
& \cdot \sum_{i=1}^{d}\left(\frac{1}{I} \int_{I}\left\|W^{-1 / p}(t) A_{I} e_{i}\right\|^{(q+\varepsilon) \alpha} d t\right)^{\frac{1}{(q+\varepsilon) \alpha}} .
\end{aligned}
$$

We can now use the fact that the function $t \rightarrow\left\|U^{1 / q}(t) x\right\|=\left\|W^{-1 / p}(t) x\right\|$ is uniformly in $A_{\infty}$ to conclude that we have a uniform inverse Hölder inequality with $\bar{q}>q$. Now choosing $\varepsilon$ and $\alpha$ to satisfy $(q+\varepsilon) \alpha<\bar{q}$ we conclude that the last integral is bounded by $\left(\frac{1}{I} \int_{I}\left\|W^{-1 / p} A_{I} e_{i}\right\|^{q} d t\right)^{1 / q}$. But $W \in A_{p}$, so $A_{I}^{-1}$ reduces averages of the metric $\rho^{*}$ given by $W^{-1 / p}=U^{1 / q}$. In other words,

$$
\left(\frac{1}{I} \int_{I}\left\|W^{-1 / p} x\right\|^{q}\right)^{1 / q} \asymp\left\|A_{I}^{-1} x\right\| \text {. }
$$

Thus finally

$$
\left\|S^{A_{I}}\left(f_{1}\right)\right\|_{L^{\alpha}\left(I, \frac{d t}{|I|}\right)} \leq C^{\prime}(\alpha, d) \inf _{t \in I}\left(M\|f\|^{p_{*}}\right)^{1 / p_{*}}(t),
$$

where $p_{*}=(p-\delta(\varepsilon)) \alpha$ is ensured to be in $(1, p)$. Similarly

$$
\left\|S^{A_{I}^{-1}}\left(g_{1}\right)\right\|_{L^{\alpha}\left(I, \frac{d t}{|I|}\right)} \leq C^{\prime}(\alpha, d) \inf _{t \in I}\left(M\|g\|^{q_{*}}\right)^{1 / q_{*}}(t)
$$

Now let us estimate $S^{A_{I}}\left(f_{2}\right)(t), S^{A_{I}^{-1}}\left(g_{2}\right)(t)$, for $t \in I$.

Let $C_{I}$ be a center of the square $Q_{I}$ built on $I$. It is easy to see that for $t \in I$ we have

$$
\left(\int_{\Gamma_{t,|I|}}\left\|\left(\nabla P_{+} A_{I} W^{-1 / p}(t) f_{2}(t)\right)(z)\right\|^{2}\right)^{1 / 2} \leq a \int P_{C_{I}}(t)\left\|A_{I} W^{-1 / p}(t) f_{2}(t)\right\| d t,
$$

where $P_{C_{I}}$ denotes the Poisson kernel. Let $\delta$ be small, $p-\delta>1$, and let $(p-\delta)^{-1}+$ $(q+\varepsilon)^{-1}=1$. We can continue the estimate as follows $\left(k_{a}^{(r) \stackrel{\text { def }}{=}}\left(1-|a|^{2}\right)^{\frac{1}{2}-\frac{1}{r}} \cdot k_{a}\right)$ :

$$
\begin{aligned}
\int P_{C_{I}}\left\|A_{I} W^{-1 / p} f_{2}\right\| \leq & \sum_{i=1}^{d} \int\left\|W^{-1 / p} A_{I} e_{i}\right\|\left\|f_{2}\right\|\left|k_{C_{I}}\right|^{2}(t) \\
\leq & C(p, q) \sum_{i=1}^{d} \int\left\|W^{-1 / p} A_{I} e_{i}\right\|\left\|f_{2}\right\| k_{C_{I}}^{(q+\varepsilon)} k_{C_{I}}^{(p-\delta)} \\
\leq & C(p, q) \sum_{i=1}^{d}\left(\int\left\|W^{-1 / p} A_{I} e_{i}\right\|^{q+\varepsilon}\left|k_{C_{I}}^{(q+\varepsilon)}\right|^{q+\varepsilon}\right)^{1 /(q+\varepsilon)} \\
& \cdot\left(\int\left\|f_{2}\right\|^{p-\delta}\left|k_{C_{I}}^{(p-\delta)}\right|^{p-\delta}\right)^{1 /(p-\delta)} .
\end{aligned}
$$

Notice that $\left|k_{C_{I}}^{(q+\varepsilon)}\right|^{q+\varepsilon} \leq C\left|k_{C_{I}}^{(q)}\right|^{q} \stackrel{\text { def }}{=} C k_{I, q}$. 
As $t \rightarrow\left\|W^{-1 / p}(t) x\right\|^{q}$ is uniformly $A_{q}$ weight (see Lemma 5.3) we can conclude that there exists a small $\varepsilon$ such that $t \rightarrow\left\|W^{-1 / p}(t) x\right\|^{q+\varepsilon}$ is uniformly $A_{q}$ weight by a classical inverse Hölder inequality. Lemma 5.2 claims that if $u$ is a scalar weight in $A_{q}$, then $\int u k_{I, q} \leq C \frac{1}{I} \int_{I} u$. Combining this knowledge we conclude that

$$
\begin{aligned}
& \sum_{i=1}^{d}\left(\int\left\|W^{-1 / p} A_{I} e_{i}\right\|^{q+\varepsilon}\left|k_{C_{I}}^{(q+\varepsilon)}\right|^{q+\varepsilon}\right)^{1 /(q+\varepsilon)} \\
& \quad \leq C \sum_{i=1}^{d}\left(\frac{1}{I} \int_{I}\left\|W^{-1 / p} A_{I} e_{i}\right\|^{q+\varepsilon}\right)^{1 /(q+\varepsilon)} \leq C \sum_{i=1}^{d}\left(\frac{1}{I} \int_{I}\left\|W^{-1 / p} A_{I} e_{i}\right\|^{q}\right)^{1 / q} .
\end{aligned}
$$

The last inequality is another application of inverse Hölder inequality.

Finally, using (5.6) we estimate the last sum by a constant.

Combining this with (5.7) and (5.8) we get $\left(p_{*}=p-\delta\right)$

$$
t \in I \Rightarrow S^{A_{I}}\left(f_{2}\right)(t) \leq C(p, q, d)\left(M\|f\|^{p_{*}}\right)^{1 / p_{*}}(t) .
$$

Similarly

$$
t \in I \Rightarrow S^{A_{I}^{-1}}\left(g_{2}\right)(t) \leq C(p, q, d)\left(M\|g\|^{q_{*}}\right)^{1 / q_{*}}(t) .
$$

Combining with our previous estimates for $S^{A_{I}}\left(f_{1}\right), S^{A_{I}^{-1}}\left(g_{1}\right)$ we get

$$
\begin{aligned}
\left\|S^{A_{I}}(f)\right\|_{L^{\alpha}\left(I, \frac{d t}{I T}\right)} & \leq C(p, q, \alpha, d)\left(M\|f\|^{p_{*}}\right)^{1 / p_{*}}(t), \\
\left\|S^{A_{I}^{-1}}(g)\right\|_{L^{\alpha}\left(I, \frac{d t}{I T}\right)} & \leq C(p, q, \alpha, d)\left(M\|g\|^{q_{*}}\right)^{1 / q_{*}}(t) .
\end{aligned}
$$

In particular, on a quarter of $I$

$$
\begin{aligned}
S_{I}(t) \stackrel{\text { def }}{=} & \int_{\Gamma_{t,|I|}}\left|\left(\nabla P_{+} W^{-1 / p} f(z), \nabla P_{+} W^{1 / p} g(z)\right)\right| d A(z) \\
= & \int_{\Gamma_{t,|I|}}\left|\left(A_{I} \nabla P_{+} W^{-1 / p} f, A_{I}^{-1} \nabla P_{+} W^{1 / p} g\right)\right| d A(z) \\
= & \int_{\Gamma_{t,|I|}}\left|\left(\nabla P_{+} A_{I} W^{-1 / p} f, \nabla P_{+} A_{I}^{-1} W^{1 / p} g\right)\right| d A(z) \\
\leq & S^{A_{I}}(f)(t) S^{A_{I}^{-1}}(g)(t) \leq C^{2}(p, q, \alpha, d) \\
& \cdot\left(M\|f\|^{p_{*}}\right)^{1 / p_{*}}(t)\left(M\|g\|^{q_{*}}\right)^{1 / q_{*}}(t) .
\end{aligned}
$$

For such $t, h(t) \geq|I|$, if we choose in the definition of $h$ to have $B=C^{2}(p, q, \alpha, d)$ and $p_{*}, q_{*}$ as above. Thus (5.4) is satisfied and the Theorem is proved.

\section{ACKNOWLEDGEMENTS}

The author is grateful to the participants of the Analysis Seminar at Michigan State University, and especially to Sheldon Axler, Michael Frazier, Fedja Nazarov, Serguei Treil and Dechao Zheng for fruitful discussions and constant enthusiasm. The author is also grateful to Robert Fefferman and Peter Jones for valuable discussions. 


\section{REFERENCES}

[AChS] Sh. Axler, S.-Y. A. Chang, and D. Sarason, Products of Toeplitz operators, Integral Equations Operator Theory 1 (1978), 285-309. MR 80d:47039

[Bl1] St. Bloom, A commutator theorem and weighted BMO, Trans. Amer. Math. Soc. 292 (1985), 103-122. MR 87g:42021

[B12] St. Bloom, Applications of commutator theory to weighted BMO and matrix analogs of $A_{2}$, Illinois J. Math. 33 (1989), 464-487. MR 91e:42030

[BG] D.L. Burkholder and R.F. Gundy, Distribution function inequalities for the area integrals, Studia Math. 44 (1972), 527-544. MR 49:5309

[B1] D.L. Burkholder, Explorations in martingale theory and its applications, Ecole d'Ete de Probabilites de Saint-Flour XIX-1989, 1-66, Lecture Notes in Math., 1464, Springer, Berlin, 1991. MR 92m:60037

[B2] D.L. Burkholder, A proof of Petczynśki's conjecture for the Haar system, Studia Math. 91 (1988), no. 1, 79-83. MR 89j:46026

[B3] D.L. Burkholder, Boundary value problems and sharp inequalities for martingale transforms, Ann. Probab. 12 (1984), no. 3, 647-702. MR 86b:60080

[Bu1] St. Buckley, Summation conditions on weights, Mich. Math. J. 40 (1993), 153-170. MR 94d: 42021

[Bu2] St. Buckley, Estimates for operator norms on weighted spaces and reverse Jensen inequalities, 340 (1993), 253-272. MR 94a:42011

[FJW] M. Frazier, B. Jawerth, and G. Weiss, Littlewood-Paley Theory and the Study of Function Spaces, CBMS Regional Conference Series in Mathematics, 79, 1991, 132 pp. MR 92m: 42021

[FKP] R.A. Fefferman, C.E. Kenig, and J. Pipher, The theory of weights and the Dirichlet problem for elliptic equations, Ann. of Math. 134 (1991), 65-124. MR 93h:31010

[GCRF] J. Garcia-Cuerva and J.L. Rubio de Francia, Weighted norm inequalities and related topics, North-Holland, 1985, vii+605 pp. MR 87d:42023

[Nik] N. K. Nikolskii, Treatise on the Shift Operator, Springer-Verlag, NY etc. 1986. MR 87i: 47042

[NT] F. Nazarov and S. Treil, The hunt for a Bellman function: applications to estimates of singular integral operators and to other classical problems in harmonic analysis, pp. 1-125, St. Petersburg Math. J. (to appear).

[S1] D. Sarason, Exposed points in $H^{1}$. II, Topics in operator theory: Ernst D. Hellinger memorial volume, 333-347, Oper. Theory Adv. Appl., 48, Birkhauser, Basel, 1990. MR 94a:46031

[S2] D. Sarason, Products of Toeplitz operators, Linear and Complex Analysis Problem Book 3, Part 1, ed. V.P.Havin, N.K.Nikolski, Lecture Notes in Math., 1573, pp. 318-319. MR 96c:00001a

[Si] I.B. Simonenko, Riemann's boundary value problem for $n$ pairs of functions with measurable coefficients and its applications to the study of singular integrals in $L^{p}$ spaces with weights, Soviet Math. Doklady, 2 (1961), 1391-1394. MR 29:253

[St] E. Stein, Harmonic analysis: Real-Variable Methods, Orthogonality, and Oscillatory Integrals, Princeton Univ. Press, Princeton, NJ, 1993. xiii+695 pp. MR 95c: 42002

[Str] D.W. Stroock, Probability Theory, an Analytic View, Cambridge Univ. Press, Cambridge, 1993. xvi+512 pp. MR 95f:6000

[TV1] S. Treil and A. Volberg, Wavelets and the angle between past and future, J. Funct. Anal. 143 (1997) (to appear).

[TV2] S. Treil and A. Volberg, Continuous wavelet decomposition and a vector HuntMuckenhoupt-Wheeden Theorem, Preprint, pp. 1-16, 1995; Ark. fur Mat. (to appear).

[TV3] S. Treil and A. Volberg, Completely regular multivariate processes and matrix weighted estimates, Preprint, pp.1-15, 1996.

[TVZ] S. Treil, A. Volberg, and D. Zheng, Hilbert transform, Toeplitz operators and Hankel operators, and invariant $A_{\infty}$ weights, Revista Mat. Iberoamericana (to appear).

Department of Mathematics, Michigan State University, East Lansing, Michigan 48824

E-mail address: volberg@math.msu.edu 